\title{
Sull'epistolario di Carlo Gozzi (in attesa del centenario)
}

\author{
Ricciarda Ricorda
}

Come è avvenuto già con l'epistolario di Gasparo Gozzi, che con tanta accuratezza e competenza, Fabio Soldini ha curato nel 1999, consentendoci di avere finalmente a disposizione, in una forma filologicamente corretta e nella sua completezza, un epistolario di altissima qualità, anche la pubblicazione delle Lettere di Carlo costituisce un vero e proprio avvenimento, destinato a portare un contributo determinante nel campo degli studi gozziani. Se il corpus proposto, con i suoi 197 documenti epistolari, è più smilzo di quello di Gasparo, che ammonta a 646 missive, ha però il grande pregio di essere nella quasi totalità inedito: nessuna lettera è stata infatti pubblicata da Carlo in vita ed anche gli amici prima e poi gli studiosi non si sono curati di questo aspetto, con minime eccezioni nei due secoli passati: negli ultimi 70 anni, ricorda Soldini, nessun nuovo documento epistolare gozziano è stato pubblicato.

Si tratta pertanto di un lavoro prezioso, che ha alle spalle una attenta ricognizione condotta in biblioteche ed archivi e che ha potuto avvalersi anche delle lettere presenti nel ricco archivio storico della famiglia Gozzi conservato nella villa di Visinale, di recente acquisito dalla Biblioteca Marciana di Venezia: si tratta di un fondo cospicuo, che si compone di varie migliaia di pagine autografe, in larga maggioranza di Carlo e in parte anche di Gasparo; custodito dall'ultima erede in linea diretta del fratello Almorò nella dimora di famiglia in Friuli, contiene anche carte inedite: è stato merito dello stesso Soldini averlo individuato ed averne favorito l'approdo alla biblioteca veneziana, già depositaria di altri manoscritti gozziani.

Il volume è arricchito da una puntuale cronologia, che rende agevole per il lettore la collocazione delle lettere, e da una ricostruzione accuratissima della bibliografia gozziana: particolarmente utile, quest'ultima, poiché per la prima volta viene proposto un catalogo così completo e preciso delle opere a stampa, da cui emergono, già a un primo esame, elementi di grande interesse: ad esempio, una metà delle voci della bibliografia risulta costituita da testi poetici, dei quali è fornito opportunamente anche l'indice.

L'epistolario comprende 32 destinatari, con una concentrazione massima intorno ai nomi dell'amico di una vita Innocenzo Massimo senior (56 lettere e 32 frammenti) e del figlio di lui, omonimo, e poi del fratello Almorò. Tra gli 
estremi cronologici del 1758, a cui appartiene la prima lettera, all'amico Paolo Balbi, e il 31 marzo 1806, data in cui Gozzi, a quattro giorni dalla morte, scrive ad Almorò l'ultimo, intenso e commovente messaggio ("Colto da improvviso malore, punta al petto, mi sento finire, sono pronto a tutto, vi benedico tutti», p. 270), le missive si articolano nell'arco di quasi un cinquantennio, seppure con sensibili concentrazioni e rarefazioni; quasi un centinaio di lettere appartiene al periodo compreso tra il 1784 e il 1787: sono gli anni dei drammi spagnoleschi, successivi all' affaire Gratarol, dell'avvio della fortuna del teatro gozziano in area germanofona; anni di cattiva salute, per Carlo, e poi dell'aggravarsi delle condizioni di salute e della morte, nel 1786, di Gasparo («da gran tempo io lo guardava come non esistente, tuttavia risovvenendomi ch'io gli son debitore delle traccie di quel poco che ho appreso, e sapendo che nel suo fondo era assai buon uomo, la di lui perdita aggrava di molto le mie afflizioni») (p. 271).

Come avviene nei migliori epistolari, è possibile privilegiare, tra le lettere di Carlo, l'itinerario che più interessa all'interno di una serie di percorsi promettenti: si potrà puntare sulla ricostruzione di aspetti di una vicenda esistenziale e di un profilo intellettuale, integrando e, più ancora, verificando l'immagine di sé che Carlo ha consegnato alle Memorie inutili, in considerazione della maggior «sincerità», o comunque libertà d'espressione, che dovrebbe competere alla comunicazione epistolare rispetto all'autobiografia — pur senza trascurare il condizionamento che il rapporto con il destinatario non può non esercitare anche in questo caso.

Così, si troveranno confermati alcuni degli elementi e dei caratteri rivelatisi centrali già nelle Memorie: ad esempio, si riscontrerà, sul piano della vita quotidiana, quanto Carlo fosse effettivamente impegnato nella gestione del patrimonio familiare, con il seguito di cause, litigi con gli affittuari, ecc., nel tentativo di arginarne il dissesto. Colpisce, in questa direzione, l'attenzione con cui lo scrittore, nonostante tutte le sue lamentele e le imprecazioni, cerca di tener dietro alle vicende e ai problemi di fratelli, cognate e nipoti: esemplare il caso di quello scavezzacollo di nome Carlo come lui, figlio del fratello Francesco, inconcludente studente a Padova, che ogni anno finisce per vendersi perfino i vestiti e che non frequenta le lezioni allo Studio con la giustificazione che sono una «formalità superflua, e ch'egli niente impara da' Signori Professori che vanno in Catedra solo per ambizione. Che si apprende più a leggere un buon libro, che andare a quelle sciocche lezioni» (p. 224).

Ma il burbero, solitario e sarcastico conte non pena solo per far fronte alle difficoltà economiche sue e dei suoi parenti: finisce infatti per farsi inguaiare anche da semplici conoscenti, come Giuseppe Canziani, che se ne va a Pietroburgo lasciandogli la cura dei due figli e, inoltre, l'incarico di vendere un carrozzino, vero e proprio «tormentone» che affligge Carlo per almeno due anni, dal 1785 al 1787, da quanto si evince dalle lettere ai due Innocenzo Massimo.

A questo proposito, merita di essere sottolineato come la vena ipocondriaca che attraversa tutto l'epistolario, riconosciuta del resto dallo scrittore stesso, laddove definisce le lettere che scambia con Innocenzo Massimo senior, anche 
lui abbastanza male in arnese, "Gazzette ipocondriache» (p. 153), abbia però alla sua base anche la somatizzazione di preoccupazioni molto reali e concrete, legate alle dissestate condizioni economiche, in cui versa ormai definitivamente la famiglia Gozzi nel suo complesso e di cui emblema efficacissimo sono quei «capponi tanto magri e infelici che fanno pietà», che arrivano dal Friuli al povero Carlo quando egli richiede «pollame purgato e ingrassato» (p. 250).

Dal nutrito fascicolo delle lettere ai due Innocenzo Massimo è possibile desumere anche ragguagli sulla vita veneziana del tempo, con indicazioni su usi e costumi e vere e proprie notizie di attualità: così, è possibile seguire da vicino i maneggi per maritare una "damina» o un figlio, oppure essere informati su fatti di cronaca (un incendio all'Arsenale, la clamorosa scoperta di una loggia massonica), riferiti con vivacità e brio molto prossimi a quelli che caratterizzano le pagine dedicate appunto alla cronaca da Gasparo sulla «Gazzetta veneta».

Qualche preziosa tessera l'epistolario riserva poi sul fronte dell'attività letteraria, che, come segnala puntualmente Fabio Soldini, è seguita in riferimento a tre ambiti principali: il rapporto con il pubblico; le relazioni con gli editori, indicati con termini assai pesanti («assemblea di pitocchi», "ammasso di ladroni increati»), in un'ottica che si rivela simpaticamente tendenziosa, non appena si confrontano tali affermazioni con la realtà che già il semplice elenco delle Opere a stampa: 1736-1805, compreso nel volume, testimonia. Il terzo ambito evocato nelle lettere è, naturalmente, quello del teatro, presente sia in termini referenziali che metaforici: per il secondo aspetto, il teatro come metafora, che si infiltra capillarmente anche nella scrittura epistolare di Carlo, ampia esemplificazione è fornita nell'Introduzione di Soldini; per il primo, vale la pena di segnalare qualche elemento inedito. Ad esempio, di grande interesse è la minuta di una lunga lettera di Gozzi del luglio 1778 a un destinatario ignoto - ma un "emissario» del Teatro Capranica in Roma che, progettando la messa in scena di alcune opere sue, gli aveva chiesto delucidazioni in merito all'allestimento — che contiene dettagliate indicazioni di regia per la Donna Serpente e la Punizione nel precipizio.

Infine, di particolare interesse risulta il bello scambio tra due dei più iracondi autori della letteratura settecentesca italiana, Carlo appunto e Giuseppe Baretti: in una lettera dell'aprile del 1777 Carlo informa Giuseppe, conosciuto, com'è noto, durante i suoi soggiorni veneziani, di essere in procinto di mandargli i volumi delle proprie opere, grato delle lodi delle Fiabe contenute nel suo Account of the manners and costums of Italy, scritto in risposta al libro pubblicato da Samuel Sharp al ritorno da un viaggio in Italia e fortemente critico nei confronti dei costumi del bel Paese. Prepara inoltre un testo, assai lungo, con una serie di puntalizzazioni, utili a precisare alcuni aspetti toccati dall'amico nell'Account, qualora questi si apprestasse a fornirne una ristampa: si tratta di uno scritto molto ampio e "rifinito", in cui Carlo commenta anche alcuni passi di Baretti, parla del suo rapporto con la truppa-trippa del Sacchi, ritorna sull'inimicizia solidale con Goldoni, ripercorre le proprie scelte teatrali, i «nuovi generi» cui si è dedicato, attacca giornalisti e giornaliste. 
Con Baretti, com'è noto, i rapporti, in seguito, si sarebbero complicati: il piemontese, infatti, non gli avrebbe risparmiato le critiche, in una famosa lettera al Carcano, ove si sarebbe dichiarato deluso dalla lettura dei testi, che non avrebbero retto alla prova del passaggio alla scrittura. Tuttavia, la prima presa di posizione barettiana sarebbe risultata fondamentale per Gozzi, che ancora nella Più lunga lettera di risposta che sia stata scritta, inviata da Carlo Gozzi ad un Poeta teatrale italiano de' nostri giorni, quella sorta di conclusivo bilancio storico e teorico della propria scrittura teatrale datato 1801, vi farà riferimento; Piermario Vescovo, proprio a proposito di questo scritto, ${ }^{1}$ ha richiamato a ragione l'attenzione sull'influenza giocata dalle posizioni teoriche del Baretti sulla riflessione gozziana, in rapporto sia alla valorizzazione della drammaturgia spagnola, sia all'ipotesi di una teatralità inesprimibile dai generi regolari.

In ultima analisi, dunque, è da sottoscrivere pienamente la valutazione con cui Fabio Soldini chiude la sua Introduzione: «l'integrale lettura di questo volume gozziano conferma: ricchezza di temi e notizie, acutezza di giudizio e forza di sentimenti, sostenutezza e varietà dello stile. Quanto basta per fare delle lettere un nuovo versante imprescindibile per la conoscenza di Carlo Gozzi e della sua scrittura» (p. 14).

1. P. Vescovo, "La più lunga lettera di risposta che sia stata scritta...». Riflessioni sull'ultimo Gozzi, in Carlo Gozzi. Letteratura e musica, a cura di B. Guthmüller e W. Osthoff, Roma: Bulzoni, 1997, p. 119-142. 